Research Article

\title{
A Clinical Pharmacological Study of the Prevalent Prescription Patterns of Metformin, Sitagliptin and Gemigliptin among the Early Moderate Grade New Type II Diabetes Mellitus Patients in Global Tertiary Care Hospitals
}

\section{Dr. Moumita Hazra*}

Medical Director, Medical Superintendent, Laboratory Director, Medical Academics and Research Director, Consultant Clinical Pharmacological Physician, Consultant Clinical Pathologist, Consultant Drug Quality and Safety Physician, Pharmaco-Haemo-MaterioVigilance Specialist, Dr. Moumita Hazra's Polyclinic And Diagnostic Centre, Dr. Moumita Hazra's Academic Centre, Dr. Moumita Hazra's Educational Centre, Hazra Nursing Home, West Bengal, India, World;

Associate Professor, Department of Pharmacology, Rama Medical College Hospital and Research Centre, Uttar Pradesh, India; Former Tutor and Post-Graduate Student, Departments of Pharmacology and Clinical Pathology, J.J.M. Medical College and Hospital, Karnataka, India;

Former Assistant Professor, Department of Pharmacology, K. D. Medical College, Hospital and Research Center, Uttar Pradesh, Hi-Tech Medical College and Hospital, Odisha, India.

*Corresponding author's E-mail: drmoumitahazra.198017thjune@gmail.com

Received: 18-06-2021; Revised: 21-08-2021; Accepted: 28-08-2021; Published on: 15-09-2021.

\section{ABSTRACT}

Type II diabetes mellitus is a common global hormonal disorder. Inhibition of dipeptidyl peptidase -4 by dipeptidyl peptidase -4 inhibitors enhances hormonal activity of incretins, like GLP - 1, GIP, GRP, stimulates insulin release and reduces glucagon secretion, finally producing anti-hyperglycaemic activity in diabetic patients. A clinical pharmacological study of the prevalent prescription patterns of metformin, sitagliptin and gemigliptin among the early moderate grade new type II diabetes mellitus patients in global multi-centre tertiary care hospitals. 100 new early moderate grade type II diabetes mellitus patients, were prescribed oral metformin $500 \mathrm{mg}$ once daily, sitagliptin $25 \mathrm{mg}$ once daily or gemigliptin $25 \mathrm{mg}$ once daily for 3 months, in monotherapy, or in combination therapy, or in a mixed regimen of monotherapy and combination therapy. The safety and efficacy assessments, with blood sugar and $\mathrm{HbA1c}$ levels and urine examination, at subsequent intervals and follow-up, were recorded and analysed. The number of prescriptions for each drug was recorded, and the respective prescription rates were statistically analysed in percentages. Metformin was most commonly prescribed ( 80 prescriptions, $80 \%$ ), followed by sitagliptin (16 prescriptions, $16 \%$ ), and gemigliptin (4 prescriptions, $4 \%$ ). Prescription frequency of metformin was followed by sitagliptin and then by gemigliptin.

Keywords: Biguanides, Metformin, Dipeptidyl peptidase-4 inhibitors, Sitagliptin, Gemigliptin, Prescription patterns.

QUICK RESPONSE CODE $\rightarrow$

DOI:

10.47583/ijpsrr.2021.v70i01.027

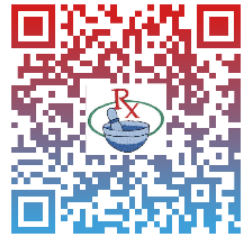

DOI link: http://dx.doi.org/10.47583/ijpsrr.2021.v70i01.027

\section{INTRODUCTION}

Type II diabetes mellitus is a very common global endocrinological disorder, witnessed in recent times. A multi-layered therapeutic approach involving a wide range of oral hypoglycaemic drugs, still remains the mainstay of type II diabetes mellitus management. The incidence and prevalence of type II diabetes mellitus (T2DM) are increasing worldwide, and the management of diabetes mellitus through advanced and effective treatment interventions is very significant in the clinical research on endocrinological pharmacology. ${ }^{1}$

The Diagnostic Criteria of type II diabetes mellitus as delineated by the American Diabetes Association include the following:
1. A fasting plasma glucose (FPG) level of $126 \mathrm{mg} / \mathrm{dl}(7.0$ $\mathrm{mmol} / \mathrm{L}$ ) or higher, or

2. A 2-hour plasma glucose level of $200 \mathrm{mg} / \mathrm{dl}$ (11.1 $\mathrm{mmol} / \mathrm{L}$ ) or higher during a 75 -g oral glucose tolerance test (OGTT), or

3. A random plasma glucose of $200 \mathrm{mg} / \mathrm{dl}(11.1 \mathrm{mmol} / \mathrm{L})$ or higher in a patient with classic symptoms of hyperglycaemia or hyperglycaemic crisis, or

4. A haemoglobin A1c (HbA1c) level of $6.5 \% \quad(48$ $\mathrm{mmol} / \mathrm{mol}$ ), or higher. ${ }^{2}$

Metformin, shows improved outcomes, as a monotherapeutic as well as a combination anti-diabetic drug, overcoming insulin resistance and lowering serum glucose levels, by the activation of $5^{\prime}$ adenosine monophosphate (AMP) activated protein kinase. Metformin is effective, safe, and inexpensive, which may reduce the risk of cardiovascular events and death. It is quite beneficial for the reduction of $\mathrm{HbA} 1 \mathrm{C}$ levels and body weight. $^{3}$

Inhibition of dipeptidyl peptidase -4 by dipeptidyl peptidase -4 inhibitors enhances the hormonal activity of 
incretins, including glucagon like peptide -1 and other bioactive peptides, (glucose-dependent insulinotropic polypeptide, and gastrin releasing peptide), thus stimulating the release of insulin and reducing the secretion of glucagons, when given in monotherapy or in combination with metformin. This effect decreases the blood glucose levels as well as HbA1c levels in type II diabetes mellitus patients, without causing severe hypoglycaemia. ${ }^{4,5}$

\section{Objective}

The objective was to conduct a clinical pharmacological study of the prevalent prescription patterns of metformin, sitagliptin and gemigliptin among the early moderate grade new type II diabetes mellitus patients in global multicentre tertiary care hospitals.

\section{MATERIALS AND METHODS}

\section{Ethical Approval}

At first, the Institutional Ethics Committee clearance and approval was taken. The study was conducted in accordance with the ethical principles of Declaration of Helsinki and Good Clinical Practices contained within the International Council for Harmonization of Technical Requirements for Pharmaceuticals for Human Use $(\mathrm{ICH}-$ E6), and in compliance with the global regulatory requirements. An informed consent was obtained from each patient participating in the study.

\section{Inclusion Criteria}

The patient inclusion criteria were as follows : (i) patients of any gender, (ii) patients within 35 and 60 years, (iii) patients presenting with new type II diabetes mellitus, of early moderate grade, (iv) type II diabetes mellitus American Diabetes Association diagnosis criteria, (v) cooperative and conscious patients, (vi) patients willing to undergo all pre and post- treatment investigations and willing to complete the entire course of treatment, (vii) patients who have given consent and are willing to go for a follow-up, (viii) patients not taking any previous antidiabetic drug, (ix) patients not taking any concomitant medication.

\section{Exclusion Criteria}

The patient exclusion criteria were as follows : (i) uncooperative or unconscious patients, (ii) patients below 35 and above 60 years, (iii) patients presenting with any grade other than early moderate grade of diabetes mellitus type II, (iv) patients with a history of hypersensitivity to any of the study drugs, ( $v$ ) patients with high risk diseases or co-morbidities, (vi) cardiac, renal or any other associated complications or co-morbidities, (vii) any chronic disease intervening with the study data, $(x)$ pregnant or lactating women, (xi) paediatric or geriatric patients, (xii) other associated medical illness or disorders, having impact on study results, (xiii) female patients using hormonal contraceptives.

\section{Study Design}

A global, multi-centre, retrospective, observational and analytical study of the clinical prescriptions of diabetic patients was performed.

\section{Study Population}

The study population consisted of 100 treated new type II diabetes mellitus patients, of early moderate grade.

\section{Study Period}

The study period, comprising of the periods for the research study and the compilation of the study literature, was 4 months, from October, 2020 to February, 2021.

\section{Place of Study}

The research study and the compilation of the study literature were done in the Departments of Pharmacology, Clinical Pharmacology, Pharmacovigilance, Internal Medicine, Endocrinology, Pathology, and Clinical Pathology, in Dr. Moumita Hazra's Polyclinic And Diagnostic Centre, Dr. Moumita Hazra's Academic Centre, Dr. Moumita Hazra's Educational Centre, Hazra Nursing Home, Rama Medical College Hospital and Research Centre, J.J.M. Medical College and Hospital, K. D. Medical College Hospital and Research Center, and Hi-Tech Medical College and Hospital.

\section{Study Procedure}

100 new early moderate grade type II diabetes mellitus patients, were prescribed oral metformin $500 \mathrm{mg}$ once daily, sitagliptin $25 \mathrm{mg}$ once daily or gemigliptin $25 \mathrm{mg}$ once daily for 3 months, in monotherapy, or in combination therapy, or in a mixed regimen of monotherapy and combination therapy.

The patients' characteristics, diabetic symptoms assessment, patients' disease and disease-related history were recorded with a proforma. Then, thorough general physical examination and systemic examination were performed on the patients under study. The relevant blood, urine and other investigations were done to confirm the progressing health status of the patients being treated.

The efficacy assessment was done, by recording the fasting and the post-prandial blood sugar level, $\mathrm{HbA} 1 \mathrm{c}$ level and urine examination findings including sugar, albumin levels and microscopy, at subsequent intervals, and at follow-up.

The safety assessment was done by the monitoring of adverse drug reactions, at subsequent intervals, and follow-up.

The prescription patterns of all the three anti-diabetic drugs were analysed. The number of prescriptions of 100 patients treated with each drug: metformin, sitagliptin, and gemigliptin was recorded; and the percentage of prescriptions for each drug was calculated. 


\section{Statistical Analysis}

The corresponding prescription rates were statistically analysed in percentages, with illustrative representation.

\section{RESULTS}

The demographic characteristics of the patients were comparable.

\section{NUMBER \% OF PRESCRIPTIONS}

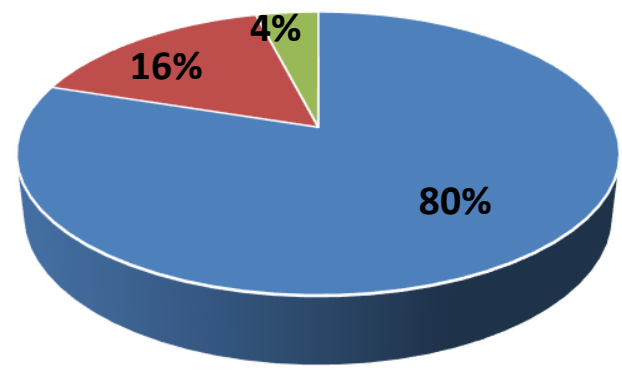

\section{- Metformin $\approx$ Sitagliptin $\approx$ Gemigliptin}

Figure 1: The Prescription Rates of Different Anti-diabetic Drugs in Percentages.

Figure 1 depicts that metformin was most commonly prescribed ( 80 prescriptions, $80 \%$ ), followed by sitagliptin (16 prescriptions, 16\%), and gemigliptin (4 prescriptions, $4 \%)$.

The prescription rates of anti-diabetic drugs were as follows: metformin > sitagliptin > gemigliptin.

The monotherapy, or combination therapy, or mixed regimen of monotherapy and combination therapy of metformin, sitagliptin or gemigliptin, was observed to be quite efficacious, which had controlled type II diabetes mellitus among new patients, with significant decrease in the blood sugar levels and the HbA1c levels, in the successive 3 months. The adverse effects observed with monotherapy, or combination therapy, or mixed regimen of monotherapy and combination therapy, were statistically non-significant. Therefore, the monotherapy, or combination therapy, or mixed regimen of monotherapy and combination therapy, were safe and tolerable.

\section{DISCUSSION}

Diabetes among Asian populations has some distinguishing characteristics from other races in the world, namely the early decrease in beta-cell function resulting in high postprandial blood glucose and the development to chronic diabetic complications occuring at an early stage of the disease. Hence, a therapeutic agent which increases beta-cell function plays an important role in antihyperglycaemic protocols.

Nowadays, anti-dipeptidyl peptidase 4 antihyperglycemic agents have been widely used for patients with T2D under guidelines of diabetes associations and proved to be effective in the enhancement of beta-cell function via ameliorating serum incretin hormone concentrations (two major incretins, GLP-1 and glucose-dependent insulinotropic polypeptide, GIP) - an anti-beta-cell apoptosis agent. There have been two incretin-related therapies for patients with T2D, namely glucagon-like peptide-1 agonists, exedin-4 and dipeptidyl peptidase-IV inhibitor, sitagliptin. In 2009, the American Association of Clinical Endocrinologists (AACE/ACE) issued the guideline for antihyperglycemic treatment protocol which mentioned about the usage of incretin therapies as the first-line drug for newly diagnosed patients with T2D (i.e, incretin therapies could be monotherapy or in combination with other antidiabetic drugs such as biguanide, sulfonylurea, or insulin). These days, incretin therapies regarding treatment for patients with T2D have been developed on a global scale and shown positive effects on not only glycaemic control but prevention from chronic diabetic complications as well.

In a research study, the drug choice was based on the guidelines of the American Association of Clinical Endocrinologists and American College of Endocrinology (AACE/ACE 2009). Patients with T2D had low HbA1C concentrations, so sitagliptin was selected as the first choice for treatment therapy in adjunct to lifestyle modification and exercises. The primary endpoint was the change from baseline in GLP-1, HOMA2-B, HOMA2-IR, HOMA2-S after 3 months of treatment with sitagliptin. Other investigative variables consisted of FPG, lipid profile, safety laboratory measurements (urea, creatinine, ALT and AST) after 3 months of treatment with sitagliptin. After 3 months of treatment with $100 \mathrm{mg} /$ day sitagliptin, patients illustrated higher HOMA2-B, HOMA2-S and lower HOMA2$\mathrm{IR}$ to those before interventions. Sitagliptin, one of the anti-DPP4 agents, has been consistently demonstrated to have effects on beta-cell and insulin concentrations indirectly prolonging active incretins and this exhibits Lcells to secrete more GLP-1. Recently, this group of agents were approved to be a second-line therapy for patients with type 2 diabetes mellitus internationally but as recommended by AACE/ACE (2009), the anti-DPP4 agents may be used to start monotherapy for type 2 diabetes patients. One model based analysis (a placebo-controlled clinical study) found that sitagliptin improved beta-cell function relative to placebo in both fasting and postprandial states in patients with T2D. DPP4 inhibitors might induce beta cell regeneration, prevention from pancreas islet hypertrophy and insulin synthesis in vitro studies. DPP4-inhibitors also improved beta-cell function both inside and outside the setting of food consumption, but some studies found there was no change in the incretin effect. Moreover, DPP-4 inhibitors would allow beta-cells to adapt to the degree of insulin resistance and have a better response to glucose overload and as the result, they decrease the overall insulin exposure and the proinsulinto-insulin ratio.

GLP-1 is a potent insulin secretagogue that exhibits glucose dependent insulin secretion. In an in vitro study, GLP-1 was found to be capable of healing beta-cell function which 
was reduced with age due to: i) recruitment of beta-cells into a secretory mode; ii) activation of the gene for glucose sensitivity of beta-cells; and, iii) reduction of beta-cell apoptosis. Treatment of old Wistar rats with GLP-1 led to the normal insulin secretion via increases of beta-cell mass and pancreas cell proliferation. And, it was hypothesized that besides the hypoglycaemic effect of anti-DPP4 agents, it may be the increase of GLP-1 that contributed to the increase of beta-cell functions. In another study, serum GLP-1 concentrations increased sharply after treatment and regression analysis confirmed that serum GLP-1 concentrations were independent variable making a great contribution to the amelioration of insulin sensitivity and insulin resistance. It was found that there were improvements in beta cell function but there were also 4 patients who still had low beta cell functions in comparison to those in the control group.

These discordances might be due to the extreme low baseline levels of beta-cell function of these 4 patients. After the treatment, changes in beta-cell function were marginal and remained low. The data denoted that serum GLP-1 concentrations negatively and positively correlated to HOMA-IR and HOMA-S, respectively when adjusted for some related factors, like age, weight, $\mathrm{HbA} 1 \mathrm{C}$, and lipid profile, which also contributed to the improvements of beta-cell function besides effects on glucose-independent insulin secretagogue. ${ }^{[3]}$

In this study, the demographic characteristics of the patients were comparable. Metformin was most commonly prescribed ( 80 prescriptions, $80 \%$ ), followed by sitagliptin (16 prescriptions, 16\%), and gemigliptin (4 prescriptions, $4 \%$ ). The prescription rates of anti-diabetic drugs were as follows: metformin > sitagliptin > gemigliptin.

The monotherapy, or combination therapy, or mixed regimen of monotherapy and combination therapy of metformin, sitagliptin or gemigliptin, was observed to be quite efficacious, which had controlled type II diabetes mellitus among new patients, with significant decrease in the blood sugar levels and the HbA1c levels, in the successive 3 months. The adverse effects observed with monotherapy, or combination therapy, or mixed regimen of monotherapy and combination therapy, were statistically non-significant. Therefore, the monotherapy, or combination therapy, or mixed regimen of monotherapy and combination therapy, were safe and tolerable.

\section{CONCLUSIONS}

The prescription frequency of metformin was followed by sitagliptin, and then by gemigliptin.

Acknowledgements: My profound acknowledgements to the Departments of Pharmacology, Clinical Pharmacology, Pharmacovigilance, Internal Medicine, Endocrinology, Pathology, and Clinical Pathology, in Dr. Moumita Hazra's Polyclinic And Diagnostic Centre, Dr. Moumita Hazra's Academic Centre, Dr. Moumita Hazra's Educational Centre, Hazra Nursing Home, Rama Medical College Hospital and Research Centre, J.J.M. Medical College and Hospital, K. D. Medical College, Hospital and Research Center, and HiTech Medical College and Hospital, for the successful completion of this research project.

\section{REFERENCES}

1. Dharmalingam $M$, Aravind SR, Thacker $H$, Paramesh S, Mohan B, Chawla M, et al. Efficacy and Safety of Remogliflozin Etabonate, a New Sodium Glucose Co-Transporter-2 Inhibitor, in Patients with Type 2 Diabetes Mellitus: A 24-Week, Randomized, Double-Blind, Active-Controlled Trial. Drugs 2020; 80: 587-600.

2. American Diabetes Association. Classification and Diagnosis of Diabetes: Standards of Medical Care in Diabetes - 2020. Diabetes Care 2020; 43 (Suppl. 1): S14-S31.

3. American Diabetes Association. Pharmacologic Approaches to Glycaemic Treatment: Standards of Medical Care in Diabetes - 2021. Diabetes Care 2021; 44 (Suppl. 1): S111-S124.

4. Le TD, Nguyen NTP, Nguyen ST, Tran HTT, Nguyen LTH, Duong $\mathrm{HH}$, et al. Sitagliptin Increases Beta-Cell Function and Decreases Insulin Resistance in Newly Diagnosed Vietnamese Patients with Type 2 Diabetes Mellitus. Diabetes, Metabolic Syndrome and Obesity: Targets and Therapy 2020; 13: 2119-2127.

5. Solerte SB, D'Addio F, Trevisan R, Lovati E, Rossi A, Pastore I, et al. Sitagliptin Treatment at the Time of Hospitalization Was Associated with Reduced Mortality in Patients with Type 2 Diabetes and COVID19: A Multicenter, Case-Control, Retrospective, Observational Study. Diabetes Care 2020; 43: 29993006.

Source of Support: The author(s) received no financial support for the research, authorship, and/or publication of this article.

Conflict of Interest: The author(s) declared no potential conflicts of interest with respect to the research, authorship, and/or publication of this article.

For any question relates to this article, please reach us at: editor@globalresearchonline.net New manuscripts for publication can be submitted at: submit@globalresearchonline.net and submit_ijpsrr@rediffmail.com 


\begin{tabular}{|l|l|} 
Corresponding author biography: Dr. Moumita Hazra \\
\hline $\begin{array}{l}\text { MBBS (Medicine), DCP (Clinical Pathology), MD (Pharmacology, Clinical Pharmacology, } \\
\text { Therapeutics), MBA (Hospital Management), PGDCR (Clinical Research). } \\
\text { Consultant Multi-Specialist Clinical Pharmacological Physician; Consultant Clinical } \\
\text { Pathologist; Associate Professor of Pharmacology \& Clinical Pharmacology for MBBS, MD, } \\
\text { DM, Dental, MSc, PhD, Nursing, Paramedical \& Pharmacy; Medical Director; Medical } \\
\text { Academics \& Research Director; Medical Superintendent; Medical Editor, Reviewer \& } \\
\text { Author. }\end{array}$ \\
\hline
\end{tabular}

